This item was submitted to Loughborough's Research Repository by the author.

Items in Figshare are protected by copyright, with all rights reserved, unless otherwise indicated.

\title{
Chord length distribution based modeling and adaptive model predictive control of batch crystallization processes using high fidelity full population balance models
}

\section{PLEASE CITE THE PUBLISHED VERSION}

https://doi.org/10.1021/acs.iecr.7b03964

\section{PUBLISHER}

(c) American Chemical Society

\section{VERSION}

AM (Accepted Manuscript)

\section{PUBLISHER STATEMENT}

This work is made available according to the conditions of the Creative Commons Attribution-NonCommercialNoDerivatives 4.0 International (CC BY-NC-ND 4.0) licence. Full details of this licence are available at: https://creativecommons.org/licenses/by-nc-nd/4.0/

\section{LICENCE}

CC BY-NC-ND 4.0

\section{REPOSITORY RECORD}

Szilagyi, Botond, Serban P. Agachi, and Zoltan Nagy. 2018. "Chord Length Distribution Based Modeling and Adaptive Model Predictive Control of Batch Crystallization Processes Using High Fidelity Full Population Balance Models". figshare. https://hdl.handle.net/2134/36090. 


\title{
Chord length distribution based modeling and
} adaptive model predictive control of batch

\section{crystallization processes using high fidelity full}

\section{population balance models}

\author{
Botond Szilágyi ${ }^{1,3,4}$, Paul Șerban Agachi ${ }^{1,2}$, Zoltán K. Nagy ${ }^{3,4^{*}}$ \\ ${ }^{1}$ Department of Chemical Engineering, "Babes-Bolyai" University, Arany Janos Street 1, Cluj \\ Napoca 400028, Romania, \\ ${ }^{2}$ Chemical, Material and Metallurgical Engineering Department, Botswana International \\ University of Science and Technology, Palapye, Botswana \\ ${ }^{3}$ Department of Chemical Engineering, Loughborough University, Leichestershire, \\ Loughborough Le11 3TU, United Kingdom,
}

${ }^{4}$ Davidson School of Chemical Engineering, Purdue University, West Lafayette 47907-2100, USA 
Keywords: crystallization, NMPC, CLD, state estimation, optimization

\begin{abstract}
The control of batch crystallizers is an intensively investigated topic as suitable crystallizer operation can reduce considerably the downstream operation costs and produce crystals of desired properties (size, shape, purity etc.). Nevertheless, the control of crystallizers is still challenging. In this work the development of a fixed batch time full population balance model based adaptive predictive control system for cooling batch crystallizers is presented. The model equations are solved by the high resolution finite volume algorithm involving fine discretization, which provides a high fidelity, accurate solution. A physically relevant crystal size distribution (CSD) to chord length distribution (CLD) transformation is also developed making possible the direct, real-time application of the focused beam reflectance measurement (FBRM) probe in the control system. The measured CLD and concentration values are processed by the growing horizon estimator (GHE), whose roles are to estimate the un-measurable system states (CSD) and to re-adjust the kinetic parameters providing an adaptive feature for the control system. A repeated sequential optimization algorithm is developed for the nonlinear model predictive control (NMPC) optimization, enabling the reduction of sampling time to the order of minutes for the one-day long batch. According to the simulation results the strategy is highly robust to parametric plant-model mismatch and significant concentration measurement noise, providing very good control of the desired CLD.
\end{abstract}




\section{Introduction}

Numerous macroscopic properties of crystalline materials of industrial interests, such as the adsorption capacity, dissolution rate, porosity, mechanical properties etc. depend on the crystal size distribution (CSD) ${ }^{1}$. The CSD also influences the downstream operations, such as filtration, granulation, centrifugation, transportation etc. ${ }^{2}$. Consequently, the good crystallizer operation might lead to simultaneous product quality improvement and operational cost reduction. The nonlinear characteristics of nucleation and growth, as well as their sensitivity to system setup, require the application of advanced control strategies to batch crystallizers: the classical and still widely used operation mode of these systems ${ }^{3}$.

The quick spread of process analytical technologies (PAT) made possible the real time tracking of solid phase: the focused beam reflectance measurement (FBRM) records the chord length distribution (CLD), a fingerprint of crystals population. The particle vision and measurement (PVM) captures in situ images in real time, which are processed by the means of image analysis. The solute concentration is tracked with on-line spectroscopic methods, such as the Raman, infrared (IR) or UV/Vis, depending on the properties of dissolved materials and the solvent ${ }^{4}$.

Numerous control strategies applied to batch crystallizers are based on PAT tools. The direct nucleation control (DNC) is a model free control, which is based on the maintenance of the FBRM count (approximately proportional to crystal number) between the predefined limits by repeated heating-cooling stages ${ }^{5}$. The crystal number is linked directly to the mean crystal size; thus the DNC controls the mean size through the FBRM count. Another state-of-the-art model free control strategy is the supersaturation control (SSC). Its principle is to keep the concentration in the metastable zone to minimize nucleation and favor crystal growth. ${ }^{6}$ Combined techniques, such as 
in-situ seed generation by DNC then switching to SSC have also been applied. ${ }^{6,7}$ The advantages of these model free control methods are that these require minimal preliminary system information and ensure satisfactory control. However, when it comes to constraint satisfaction and optimal operation, these model free techniques can hardly compete with the predictive power of model based controllers. ${ }^{8}$

The population balance models (PBMs), introduced by Hulburt and Katz ${ }^{9}$ are widely used to describe and simulate crystallization processes ${ }^{10}$. The $\mathrm{PB}$ equation (PBE) is a hyperbolic partial differential equation (PDE). Its numerical solution is difficult as it tends to produce numerical diffusion and oscillation ${ }^{11}$. Finding analytical solutions is possible only in limited cases, which are seldom practically relevant. Numerous numerical methods have been proposed for the solution of PBE's, all with advantages and disadvantages. From the process control point of view the solution has to be fast enough to be applicable in real time optimization and detailed enough to provide the required information (for example, whole CSD or mean size) ${ }^{12}$. In order to use the (distributional) CLD in the model based control, the calculation of CSD is required, thus the numerically very efficient moment based methods ${ }^{13}$ are not applicable. The high resolution finite volume method (HR-FVM) is an efficient algorithm to compute the CSD without significant numerical diffusion and/or oscillations ${ }^{14}$. The solution speed can be improved to meet the real time applicability with efficient implementations ${ }^{15}$.

The overwhelming majority of real time controllers and process optimizations involve moment based PBE solution ${ }^{8,16-18}$ because of its decreased computational expense and high accuracy. More recent work employs machine learning to reduce the computational costs. ${ }^{19}$ However, these operate on mean crystal properties, not on the actual CSD. Among of full PBM based strategies the open loop control is most widely discussed and applied. ${ }^{20-22} \mathrm{~A}$ feedback concentration control 
system tracking the pre-computed optimal concentration trajectory based on a 2D full PBM was recently published. ${ }^{23}$ Only a few papers deal with full PBM based real time control ${ }^{24}$, where the authors concluded that the critical point in real time applicability is the proper mesh coarseness, which is the result of a trade-off between accuracy and computational burden. In the same work it was also presented that the structural model-plant mismatches (PMM) (i.e. errors related to discretization coarseness) can be effectively eliminated by output error correction. Nevertheless, the paper does not deal with parametric PMM, which raises special control problems.

Regardless of model complexity, for robust NMPC performance, state estimation is required ${ }^{25}$. State estimators of various internal structure and working principle have been developed, including the stochastic Kalman filter family, ${ }^{26,27}$ Luenberger observers, ${ }^{17,28}$ and moving horizon state estimators (MHE). ${ }^{24,29}$ The MHE is an optimization based method involving the non-linear process model and, in contrary to the other estimators, it uses measurements gathered over a certain time interval for the observer correction. ${ }^{25}$

In order to utilize the FBRM provided CLD, the CSD $\rightarrow$ CLD transformation needs to be carried out. Numerous papers have focused on the calculation of CSD from measured CLD data ${ }^{30-34}$, as the CSD is physically relevant and it is often the controlled quantity. The problem with these approaches is that none of those are exact due to the CLD multiplicity (the same chord length might be coming from more particles, depending on particle size, shape and spatial orientation). As the backward CLD $\rightarrow$ CSD transformation is often carried out by optimization, this step might be time consuming.

The objective of this study is to develop a shrinking horizon NMPC for the product CSD for fixed batch time cooling crystallization processes. The control strategy involves a growing horizon estimation (GHE) algorithm, for which the estimation horizon is growing with the actual process 
time. To deal with parametric model-plant mismatch (PMM) the GHE has the role, next to the estimation of un-measurable system states, to improve continuously the model quality by readjusting the kinetic parameters. A real time CSD $\rightarrow$ CLD forward transformation is developed, making possible the direct application of FBRM data in the CSD control system. In the NMPC calculations an accelerated direct single shooting dynamic optimization strategy is developed and applied, which reduces the calculation time to the range of feasible industrial sampling time. To avoid structural PMM, fine mesh is applied in the PBM solution. The results indicate that a high fidelity population balance model based adaptive model predictive control that uses model parameters identified directly using FBRM measurement and a novel efficient forward CSD to CLD conversion approach can be applied to batch cooling crystallization processes to directly control product consistency based on the target CLD signature.

\section{Mathematical model of the batch cooling crystallization system}

The batch operation is the classical crystallization technique, which is based on the temperature dependency of solubility. This is often modeled with a power-law equation:

$$
c_{S}(T)=p_{1}+p_{2} T+p_{3} T^{2}
$$

where $c_{S}$ denotes the solubility concentration, $T$ is the temperature and $p_{i}$ are material and system dependent constants.

The initially high temperature, concentrated solution is cooled. With cooling, the solubility decreases and the solution becomes supersaturated. The supersaturation is characterized by the relative supersaturation:

$$
\sigma=\frac{c}{c_{S}(T)}-1
$$


where $c$ denotes the actual solute concentration. The supersaturation generates automatically spontaneous crystal nucleation. Most often being the governing mechanism in industrial crystallizers, in this study secondary nucleation is considered:

$$
B(\sigma, T)=k_{b} \sigma^{b} V_{C}^{j} \exp \left[-\frac{E_{b}}{R T}\right]
$$

where $V_{C}$ is the volume fraction of crystals, $R$ is the gas constant and $k_{b}, b, j$ and $E_{b}$ are material and system specific constants.

The nuclei and existing crystals are growing in the supersaturated solution with the growth rate:

$$
G(\sigma, T)=k_{G} \sigma^{g} \exp \left[-\frac{E_{g}}{R T}\right]
$$

where $k_{g}, g$ and $E_{g}$ are material and system specific constants. A cooling batch crystallizer with nucleation and growth sub-processes is illustrated in Figure 1.

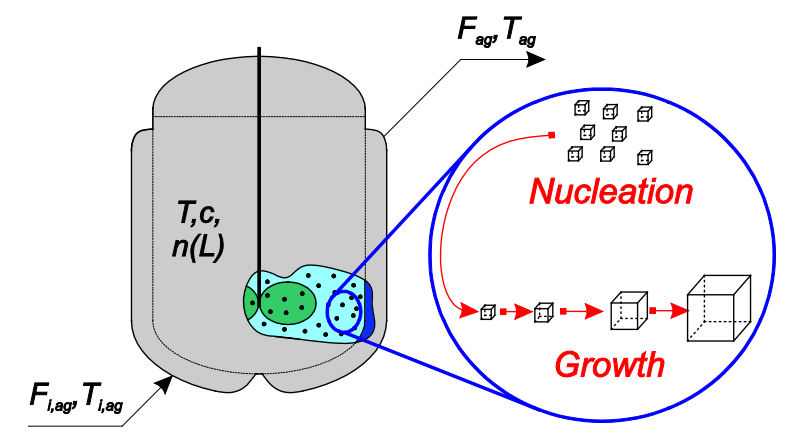

Figure 1. Schematic representation of a cooling batch crystallizer with nucleation and growth mechanisms.

For the characterization of the CSD the uni-variate size density function $n(L, t) d L$, which gives the number of crystals within the $L$ and $L+d L$ size domain at $t$ time moment. The variation of CSD under the influence of nucleation and growth is governed by the PBE:

$$
\frac{\partial n(L, t)}{\partial t}+G(\sigma, T) \frac{\partial n(L, t)}{\partial L}=B(\sigma, T) \delta\left(L-L_{n}\right)
$$


with $n(L, t)=n_{0}(L)$ initial condition, where $n_{0}(L)$ represents the seed distribution and $L_{n}$ is the nuclei size. In this study a log-normally distributed seed population is considered.

Due to the liquid-solid mass transfer a macroscopic mass balance is required for solute concentration:

$$
\frac{d c}{d t}=-\frac{k_{V} \rho_{c}}{1-V_{C}}\left[3 G(\sigma, T) \int_{0}^{L_{\max }} L^{2} n(L, t) d L+B(\sigma, T) L_{n}^{3}\right]
$$

with $c(0)=c_{0}=c_{s}\left(T_{0}\right)$ initial concentration. In Eq.(6) $\rho_{c}$ is crystal density and $k_{V}$ is the volume shape factor:

$$
v_{c}=k_{V} L^{3}
$$

The temperature is a manipulated variable thus energy balance is not required for the model closure. The values of process and kinetic parameters are listed in Table 1. The solubility refers to L-ascorbic acid and the kinetic parameters are partial results of L-ascorbic acid parameter estimation. A detailed study on L-ascorbic acid crystallization and control will be presented elsewhere.

Table 1. Process and kinetic parameters used in the simulation studies.

\begin{tabular}{cll}
\hline Parameter & Name & Value, U.M. \\
\hline$p_{1}$ & Solubility constant & $0.1416, \mathrm{~g} \mathrm{~g}^{-1}$ \\
$p_{2}$ & Solubility constant & $3.3710^{-3}, \mathrm{~g} \mathrm{~g}^{-1} \mathrm{o}^{-1}$ \\
$p_{3}$ & Solubility constant & $4.0210^{-5}, \mathrm{~g} \mathrm{~g}^{-1} \mathrm{O}^{-2}$ \\
$k_{b}$ & Nucleation rate constant & $1.2410^{17}, \# \mathrm{~m}^{-3} \mathrm{~s}^{-1}$ \\
$b$ & Nucleation rate supersaturation exponent & $1.377,-$ \\
$j$ & Nucleation rate volume fraction exponent & $0.980,-$ \\
$E_{b}$ & Nucleation activation energy & $39760, \mathrm{~J} \mathrm{~mol}^{-1} \mathrm{~K}^{-1}$ \\
$k_{g}$ & Growth rate constant & $11266, \mu \mathrm{m} \mathrm{s}^{-1}$ \\
\hline
\end{tabular}




\begin{tabular}{cll}
\hline$g$ & Growth rate supersaturation exponent & $0.565,-$ \\
$E_{g}$ & Growth activation energy & $34939, \mathrm{~J} \mathrm{~mol}^{-1} \mathrm{~K}^{-1}$ \\
$\rho_{c}$ & Crystal density & $1650 \mathrm{~kb} \mathrm{~m}^{-3}$ \\
$k_{V}$ & Volume shape factor & $1,-$ \\
$T_{\text {in }}$ & Initial temperature & $34.5,{ }^{\circ} \mathrm{C}$ \\
$m$ & Mean of (seed) log-normal distribution & $20, \mu \mathrm{m}$ \\
$v$ & Variance of (seed) log-normal distribution & $10, \mu \mathrm{m}$ \\
$V_{C, \text { seed }}$ & Seed volume fraction & $4.8910^{-4},-$ \\
$L_{n}$ & Nucleon size & $10^{-6} \mathrm{~m}$ \\
\hline
\end{tabular}

The model equations were solved by using an efficient implementation of a fully discretized HR-FVM, whose description, due to brevity, is not repeated here ${ }^{35}$. The in-house developed crysiv MatLab based toolbox was used to solve the model equations, implemented as compiled serial C .mex function ${ }^{15}$. Based on the results of preliminary experiments in the model solution the 0-1000 $\mu \mathrm{m}$ size domain was chosen. The uniformly distributed mesh with $\mathrm{N}=1000$ elements ensured accurate model solution.

According to the model Eqs.(1)-(7) the product CSD depends on initial conditions, the vector of kinetic parameters and the applied temperature profile:

$$
C S D_{\text {prod }}=f\left(T_{0}, n_{0}, K P, T(t)\right),
$$

where the vector of kinetic parameters $(K P)$ is defined as:

$$
K P=\left[k_{b}, b, j, E_{b}, k_{g}, g, E_{g}\right]
$$

Under given conditions $K P$ is constant but might be sensitive to system setup (e.g. crystallizer shape, volume, etc.), impurities and operational conditions (e.g. stirrer type and revolution speed, hydrodynamic conditions etc.). In this study $K P$ is not considered constant, but it is re-estimated 
during the process, which also incorporates the effects of disturbances, and provides an adaptive feature of the control algorithm, providing very high robustness to the feedback control approach.

\section{FBRM soft sensor model forward transformation of crystal size distribution to chord length distribution}

The mathematical model presented in the previous section enables the calculation of the evolution of the CSD and solute concentration. There are numerous PAT tools to track the solute concentration (e.g. spectroscopic techniques based on ATR-IR, UV-VIS, Raman) but the CSD is difficult to be measured with on-line techniques. The CLD provided by the FBRM is related to the CSD, although it does not reflect the true CSD. As a consequence, a transformation is required between these distributional quantities.

The measuring principle of FBRM is presented in Figure 2. The probe emits a rotating laser beam. During the rotation the beam intersects with the particles during which the laser beam is reflected back into the probe's detector. The intersection time is recorded and in the knowledge of laser rotation speed the intersection length, the so-called chord length (CL) is calculated. The typical number of intersections, often referred as FBRM counts, is in order of thousands per second. From the individually measured CLs, a CL distribution (CLD) can be constructed $n(C L, t) d C L$, which gives the number of $C L$ s within the $C L, C L+d C L$ domain at $t$ time moment.

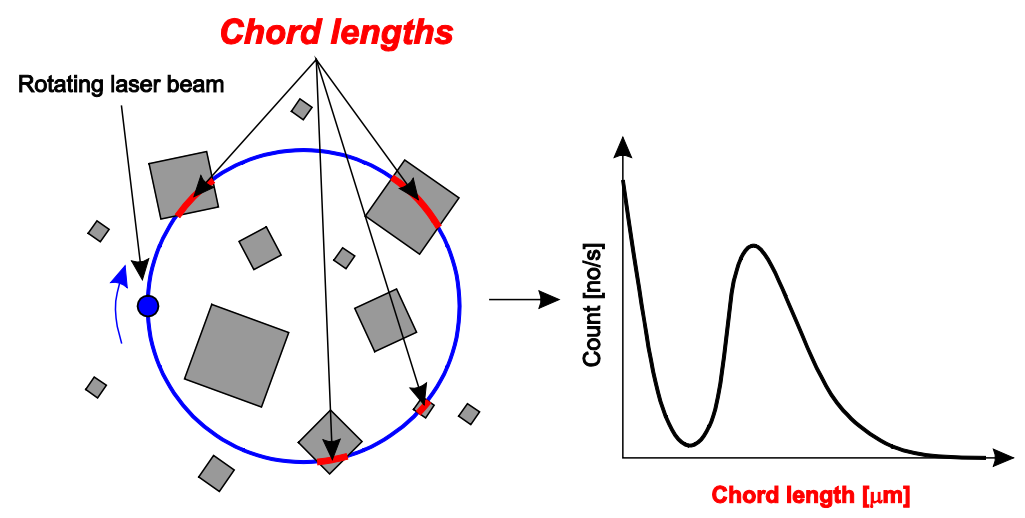


Figure 2. Working principle of the FBRM and the construction of CLD.

Here a straightforward CSD $\rightarrow$ CLD transformation is developed. According to Figure 3, the 2D projection of a particle (detectable by the FBRM) can give a variety of CLs, depending on how the particle is intersected by the beam. In this context a CLD can be constructed for each 2D projection of the same cube (or any other regular shape).

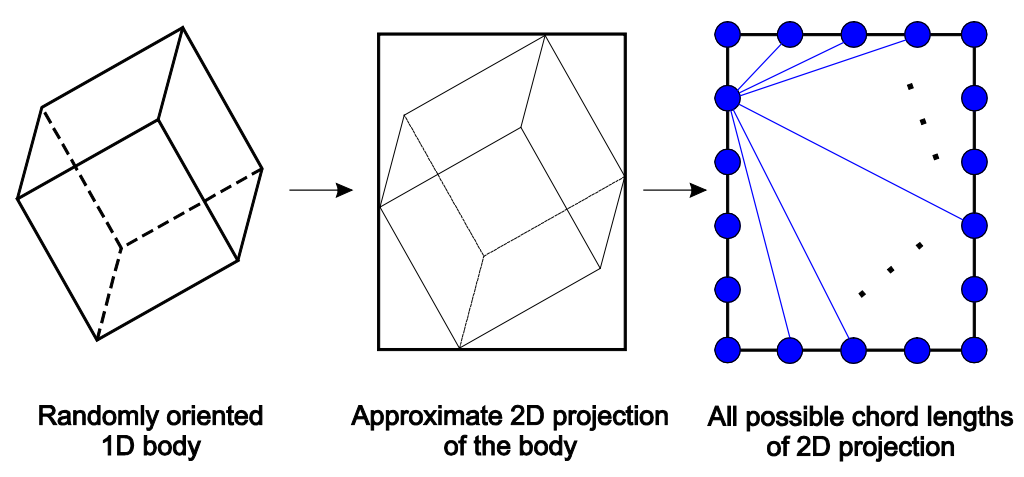

Figure 3. Schematic representation of the projection-based CLD simulation

The basic idea of the proposed projection based CLD calculation is:

- Take a random orientation of the cubic particle,

- Create the 2D projection of cubic particle taking into consideration the assumed spatial orientation,

- Put the 2D projection into a rectangle,

- From the possible "cuts" calculate the $\mathrm{CLD}_{\{\text {proj }\}}$ of this projection,

- Repeat the calculation for all possible projections and average the results.

The CLD simulation of a single particle (or mono-disperse particle population) can be carried out by this method. Assuming that the cube is projected into horizontal plane, two angles are required to define unambiguously its spatial orientation: the relative angle to the horizontal $(\alpha)$ and 
to one of the vertical $(\beta)$ planes. If both $\alpha$ and $\beta$ goes from $0^{\circ}$ to $90^{\circ}$, taking into consideration the cube shape, all possible spatial orientations are covered. In the algorithm these two angles are varied and for each $\{\alpha, \beta\}$ projection the $C L D_{\{\alpha, \beta\}}$ is simulated. After all $C L D_{\{\alpha, \beta\}}$ are computed based on the individual projections, these are averaged, which results in the most probable CLD of mono-disperse particle population of $L$ size.

In these simulations both angles were varied with an increment of $3^{\circ}$. Thus, the angles are considered to be uniform random variables. The explanation of this choice is that since the crystals are stout, there is no reason why they would follow any organized trajectory with preferred orientation that would require the use of a non-uniform distribution. Using this discretization in the angles the variations in the final CLD were negligible, thus the $3^{\circ}$ value is used for improving the simulation speed. The simulated $C L D$ of the cubic particle having $L$ linear size, $C L D_{L}$ is given by

$$
C L D_{L}=\frac{\sum_{j=1}^{P} \operatorname{CLD}_{\{j\}, i}}{\sum_{j=1}^{P} \sum_{i=1}^{M} \operatorname{CLD}_{\{j\}, i}}
$$

In Eq.(10) $P$ is the number of individual $C L D_{\{\alpha, \beta\}}$ and $M$ denotes the number of discrete CL size bins. In this study logarithmic CL scale is adopted with [1..1000] $\mu \mathrm{m}$ domain and 90 bins. The simulation is implemented as compiled serial C .mex function involving single precision floating point operations.

All $C L D_{L S}$ are pre-computed for each discrete particle size used in the HR-FVM solution of the PBE. The most probable $C L D$ corresponding to the simulated $C S D$ is computed in real time as the normalized weighted sum of the individual $C L D_{L} \mathrm{~s}$ :

$$
C L D=\frac{\sum_{j=1}^{N} A_{L} n_{L} \operatorname{CLD}_{\mathrm{L}, i}}{\sum_{j=1}^{N} \sum_{i=1}^{M} \operatorname{CLD}_{\mathrm{L}, i}}
$$


where $N$ denotes the HR-FVM mesh size, $n_{L}$ stands for the number of crystals with size $L$ and $A_{L}$ is the most probable projection area of the particle. With $A_{L}$, the transformation takes into consideration that the bigger crystals are captured with higher probability. The execution time of Eq. (11), implemented as compiled serial C .mex function is in order of milliseconds. The simulated CLD given by Eq.(11) should be compared to normalized measured CLD.

For comparing purposes, the CLD simulation of spherical crystals has also been carried out, which is significantly easier since every $2 \mathrm{D}$ projection is a circle with the same diameter. This CLD transformation has already been carried out and analyzed in the literature ${ }^{36}$ thus, for brevity, the description will not be repeated here.

To illustrate the CSD $\rightarrow$ CLD transformation approach, a CSD is assumed as the sum of two lognormal distributions, and the CLD is simulated based on the aforementioned method, assuming both spherical and cubic shapes. Together with the original CSD and simulated CLDs, the volume based CSD is also represented in Figure 4, as this is used often for the characterization of the particle population. It can be observed that the simulated CLD for cubic crystals correlates very well with the volume based CSD, for which the CLD was simulated. This might be because there are many CLs in a cube that are longer than the actual edge length. It can also be seen that assuming cubic shape for the same CSD generates significantly larger CLs than the spherical shape. The reason for this is that the largest diagonal of the cube is $L \sqrt{3}$, which inherently generates offset between the CLD estimated based on cubic and spherical shapes. 


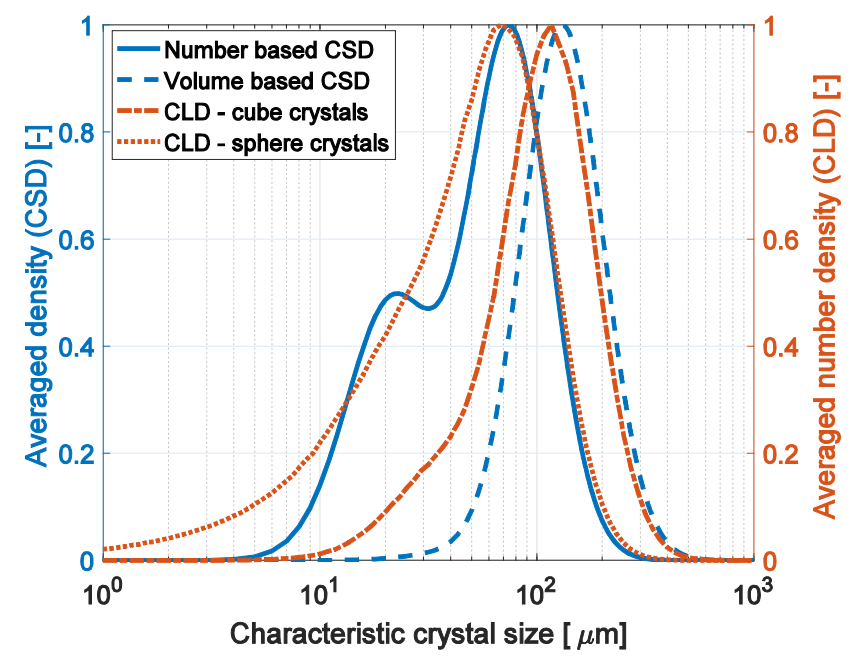

Figure 4. Crystal size distributions (CSD) and the corresponding simulated chord length distributions (CLD).

Figure 5 evaluates the performance of the CSD to CLD transformation by comparing its results with the experimental CLD of a known CSD. The ceramic bead particles, reported in literature, have nearly ideal spherical shape and the CSD was calculated by image analysis. ${ }^{33}$ The simulated CLD agrees acceptably with the measured CLD reported in literature. ${ }^{33}$ This justifies the application of the proposed transformation for process control purposes, which also relies on the auto-corrective feature provided by the feedback measurement.

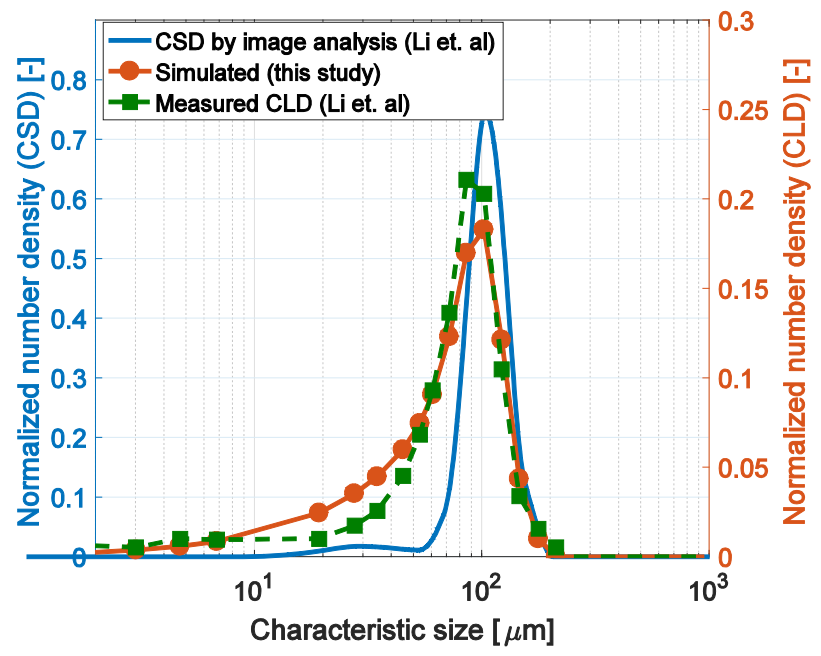


Figure 5. Comparison of CLD transformation with experimental data available from literature for spherical particles.

The applied forward CSD $\rightarrow$ CSD transformation might not be reliable for two situations:

- For dense suspensions with high crystals concentration (i.e. intensive nucleation) the fine particles might overlap and higher apparent CLs might be measured than expected.

- $\quad$ For the largest crystals of which size is comparable with the beam rotation diameter (8 $\mathrm{mm}),{ }^{37}$ the approximation of $\mathrm{CL}$ with a straight line might introduce distortions.

The first effect is an interaction effect, which increases the apparent CL especially in the low CL domain. The second effect results from a modelling simplification for very large crystals, which are outside of the interest of general CSD control problems.

In this work cubic shape is assumed, for which the CLD calculation is computationally more expensive.

\section{Open loop temperature optimization}

The open loop model based control consists of the pre-calculation of the optimal temperature profile, which is tracked by a regular PI(D) controller. For the optimization a target CSD is required within the achievable domain. The target CSD is generated by simulating a ten-day long batch, applying linear cooling between the initial and final $\left(\mathrm{T}=20^{\circ} \mathrm{C}\right)$ temperatures. This leads to a conservative target CLD, which requires the controller to find an optimal profile that avoids/minimizes nucleation, while maintaining yield, when the duration of the batch is decreased.

In the optimization the batch time is evenly divided into $K$ discrete intervals, generating the vector of discrete time moments between the initial $\left(t_{0}=0\right)$ and final $\left(t=t_{K}\right)$ time moments: $\left(t_{v}=\left[0, t_{1}, \ldots, t_{K}\right]\right)$. This defines the optimization horizon, which is extended with an additional time interval, required for system equilibration $\left(t_{K+1}=t_{K}+t_{e q}\right)$. The temperature profile vector 
$\left(T_{v}\right)$ is the vector that contains the temperatures corresponding to $t_{v}$ time moments. During the equilibration period $\left(t_{K}<t<t_{K+1}\right)$ the last temperature is kept constant $\left(T_{K+1}=T_{k}\right)$.

Then, the temperature profile optimization is carried out subject to a sum-square error based objective function:

$$
\operatorname{SSE}\left(T_{V}\right)=\sum_{i=1}^{U}\left(D_{i, s i m}-D_{i, r e f}\right)^{2}
$$

In this optimization the 24 hours batch time is divided to $K=400$ intervals thus $T_{V}$ is a vector of 400 elements. $D$ denotes the distributional data (CSD or CLD) based on which the temperature profile optimization is carried out. $U$ stands for the number of discrete bins in $D$. The optimization is carried out subject to the following constraints:

$$
\begin{aligned}
& 18 \leq T_{K} \leq 24{ }^{\circ} \mathrm{C} \\
& 0.01 \leq c_{r} \leq 0.5{ }^{\circ} \mathrm{C} / \mathrm{min} \\
& t_{e q}=7200 \mathrm{~s}
\end{aligned}
$$

where $c_{r}$ is the cooling rate. The constrained optimization was carried out in MatLab environment using the patternsearch built-in optimization function, which uses the pattern search algorithm ${ }^{38}$.

In Figure 6a the optimal temperature profiles are presented. As the higher degree weighted CLD is used as the target, the parabolic characteristics of the temperature profile becomes more dominant. The Figure $6 \mathrm{~b}$ presents the target as well as the realized CSD's. The CSD based optimization approached the most the target, the CLD based optimizations leads to more significant fine production. In general, the parabolic temperature profiles are known to be the optimal for nucleation rate minimization. ${ }^{39}$ 


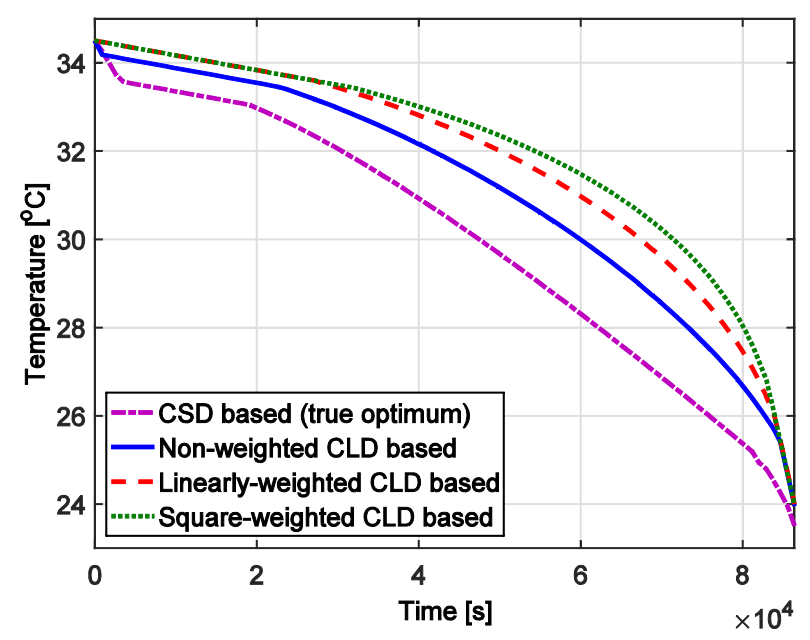

a)

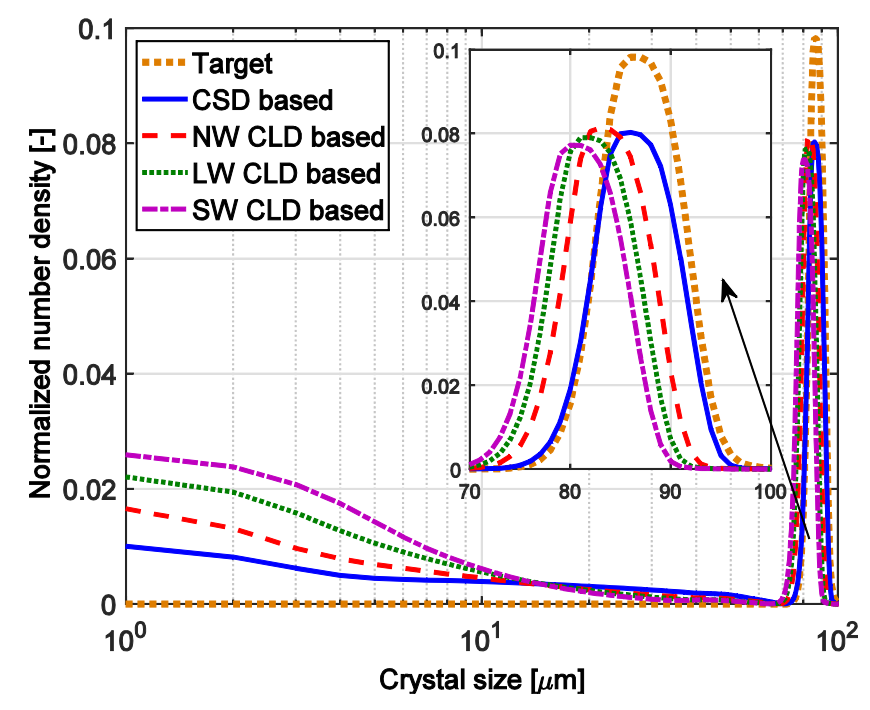

b)

Figure 6. a) The optimal temperature profiles based on CSD and differently weighted CLD b) The target and product CSD's realized by the temperature profiles of Figure 6a).

According to the results the non-weighted CLD is the best option to use in process control. This is because it introduces less distortion in small crystal size range so it reflects the nucleation more sensitively. 


\section{The growing horizon estimation (GHE)}

For an NMPC approach to work robustly in practice state or error estimation is required, which calculates the actual values of measurable and un-measurable system states providing the initial conditions of the NMPC optimization. Estimation can be performed using a receding (moving) or a growing horizon. While for continuous processes only receding horizon estimation can be implemented for batch systems both receding horizon estimation (RHE) and GHE is possible. Both the RHE and GHE use the non-linear process model thus present increased potential in dealing with process non-linearities ${ }^{25}$. The main advantage of GHE is that the initial system states (solute concentration and CSD) are generally accurate for batch system, and often the states at time zero are better known than at any subsequent time steps. For example, in the case of an unseeded batch crystallization the moments of the distribution are well known at time zero (all are zero). As a consequence, a well-tuned GHE might provide more accurate states for the NMPC optimizations than a RHE approach which might be based on measurement or previously estimated initial state estimates. Nevertheless, this state estimation is difficult during the primary nucleation, which produces crystals that are comparable in size with the detection limit of PAT tools and also has negligible impact on the solute concentration. This estimation uncertainty can be moderated by fine-tuning the state-estimator and by having good initial kinetic parameters. After the nucleation, the reliability of the state estimation increases from iteration to iteration as more and more measurement data become available.

Modifications in operating conditions, system set-up or the presence of impurities can lead to variation in the nucleation and/or growth rates, which might lead to considerably different macroscopic behavior, thus the system might require substantially different control policy. If these changes occur during the crystallization process the model parameters need to be updated and the 
control policy recalculated for best performance. The GHE re-adjusts the kinetic parameters in every sampling time by fitting the model to existing measured data.

The control system block diagram is presented in Figure 7a. The input of the GHE is the measured data, based on which it re-adjusts the kinetic parameters as well as calculates the actual system states. Figure $7 \mathrm{~b}$ shows the general growing/shrinking horizon concept of a batch GHENMPC considering a fixed batch time.

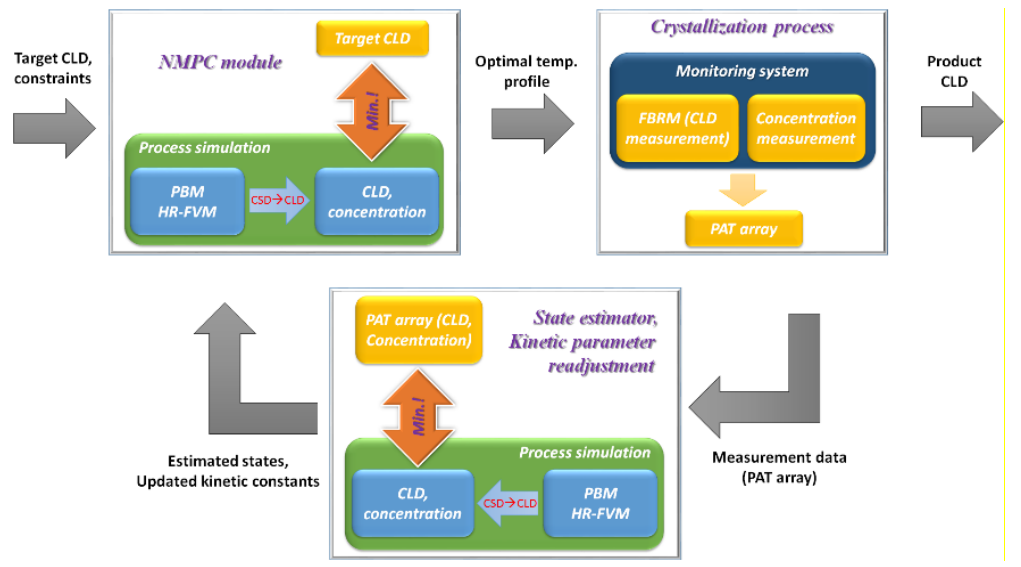

a)

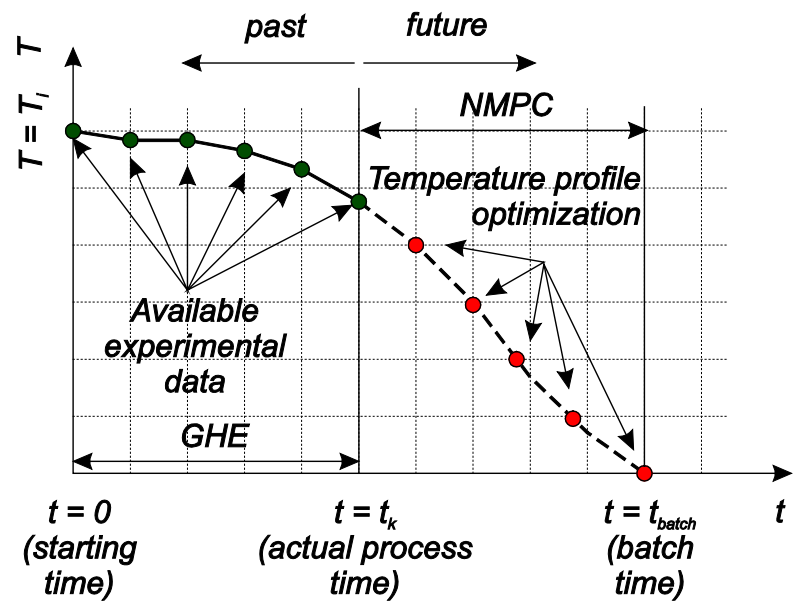

b)

Figure 7. a) Block diagram of the adaptive/predictive model based control system and b) working principle of fixed batch time GHE/NMPC. 
The GHE uses the same non-linear process model as the NMPC but the nucleation Eq.(3) and growth Eq.(4) rates are expressed differently:

$$
\begin{aligned}
& B(\sigma, T)=10^{k_{b, G H E}} \sigma^{b} V_{C}^{j} \exp \left[-\frac{E_{b}}{R T}\right] \\
& G(\sigma, T)=10^{k_{g, G H E}} \sigma^{g} \exp \left[-\frac{E_{g}}{R T}\right]
\end{aligned}
$$

The reason for using modified rate equations in parameter readjustment is to improve the optimization performance: in Eq.(14)-(15) the rate constants are exponents (as the rest of kinetic parameters), which acts like a natural scaling: minor variations in the value of these modified rate constants influence substantially stronger the growth rates.

The modified vector of kinetic parameters takes the form:

$$
K P_{R H E}=\left[k_{b, G H E}, b, j, E_{b}, k_{g, G H E}, g, E_{g}\right]
$$

The GHE uses the following objective function:

$$
\operatorname{SSE}\left(K P_{G H E}\right)=\sum_{i=1}^{Z}\left(c_{i, \exp }-c_{i, \text { sim }}\right)^{2}+w \sum_{j=1}^{M} \sum_{i=1}^{Z}\left(C L D_{i, j, \exp }-C L D_{i, j, s i m}\right)^{2}
$$

The first part of the objective function minimizes the difference between the simulated and measured concentrations, whereas the second part of the objective minimizes the deviation of the simulated from the measured CLD. $w$ is a weighting factor; $Z$ denotes the number of discrete time moments in which measurement data is available or is used in the state estimation. The optimization was carried out by using the fmincon MatLab function with the interior point algorithm.

To improve the GHE calculation time, the maximum number of used estimation points is limited to $N_{\text {est,max }}$. If the number of available measurements exceeds $N_{e s t, m a x}$, instead of switching to a RHE approach, a new uniformly distributed time vector is defined in $\left[0, t_{k}\right]$ interval with $N_{e s t, \max }$ 
data points. Thus, the time step size $\left(\Delta t_{e}\right)$ in the estimation horizon is calculated, in the knowledge of process sampling time $(\delta t)$ as follows:

$$
\Delta t_{e}=\left\{\begin{array}{c}
\delta t, \text { ift } t_{k} \leq N_{\text {est }, \max } \delta t \\
\frac{t_{k}}{N_{\text {est,max }}}, \text { otherwise }
\end{array}\right.
$$

If needed the concentrations and CLD data are interpolated from the measured data in the $\Delta t_{e}$ time steps using the measurement data available in $\delta t$ time steps. A piecewise cubic Hermite interpolation was used by calling the pchip MatLab function. With this approach the advantage of the GHE of using the known initial states at the start of the batch is still maintained without significant increase in the computational burden due to excessive increase in the number of measurement points in the growing horizon. The most important GHE settings and tuning parameters are listed in Table 2.

Table 2. GHE tuning parameters

\begin{tabular}{cll}
\hline Parameter & Name & Value, U.M. \\
\hline$N_{\text {est,max }}$ & Maximum number of time moments & $40,-$ \\
$N_{\text {est }, \text { iter }}$ & Maximum number of optimization iterations & $130,-$ \\
$w$ & Weighting parameter in objective function & $10^{-4},-$ \\
\hline
\end{tabular}

To make the parameter estimation more efficient for real-time feasibility the kinetic parameters obtained in the previous estimation step are used as the initial guess for the next optimization problem. The first GHE problem uses the kinetic parameters obtained from off-line model identification performed prior to the control experiment. The decision variables $\left(K P_{R H E}\right)$ are bounded. Choosing the correct bounds is essential: if the search interval is too narrow the identification may fail to identify new set of parameters when larger disturbance or change in 
process occurs, but too wide intervals might be harmful especially in the first iterations, due to the nucleation-generated fine crystals, which are notoriously difficult to measure correctly as they have reduced effect on solute concentration and are close or below the detection limit of the PAT tool. The applied bounds are listed in Table 3. These can be considered as additional GHE tuning parameters.

Table 3. Bounds of decision variables in GHE kinetic parameter re-adjustment

\begin{tabular}{cll}
\hline Parameter & Lower bound [\% to actual] & Upper bound [\% to actual] \\
\hline$k_{b, G H E}$ & -20 & +20 \\
$b$ & -6 & +6 \\
$j$ & -2 & +2 \\
$E_{b}$ & -8 & +8 \\
$k_{g, G H E}$ & -20 & +20 \\
$g$ & -6 & +6 \\
$E_{g}$ & -8 & +8 \\
\hline
\end{tabular}

In order to examine the performance of the developed state estimator a test simulation was carried out in which it was assumed that the initial parameter estimates in the model differed from the actual kinetic parameter of the process (Plant). The parameters in "Plant" and "Model" simulation are listed in Table 4. 
Table 4. The Plant and the (initial) Model parameters

\begin{tabular}{cll}
\hline Parameter & Plant & Model \\
\hline$k_{b}$ & $1.2410^{17}, \# \mathrm{~m}^{-3} \mathrm{~s}^{-1}$ & $9.9310^{16}, \# \mathrm{~m}^{-3} \mathrm{~s}^{-1}$ \\
$b$ & $1.377,-$ & $1.65,-$ \\
$j$ & $0.980,-$ & $0.882,-$ \\
$E_{b}$ & $39760, \mathrm{~J} \mathrm{~mol}^{-1} \mathrm{~K}^{-1}$ & $33796, \mathrm{~J} \mathrm{~mol}^{-1} \mathrm{~K}^{-1}$ \\
$k_{g}$ & $11266, \mu \mathrm{m} \mathrm{s}^{-1}$ & $7886, \mu \mathrm{m} \mathrm{s}^{-1}$ \\
$g$ & $0.565,-$ & $0.621,-$ \\
$E_{g}$ & $34939, \mathrm{~J} \mathrm{~mol}^{-1} \mathrm{~K}^{-1}$ & $40180, \mathrm{~J} \mathrm{~mol}^{-1} \mathrm{~K}^{-1}$ \\
\hline
\end{tabular}

In the temperature profile optimization the objective function Eq.(12) subject to the constraints Eq.(13) was applied and the optimization was solved using the pattern search algorithm (patternsearch Matlab function) ${ }^{38}$.

Figure 8a presents four temperature profiles. The "Plant optimum" is the optimal temperature profile calculated by CSD based optimization with the "Plant parameters". This strategy operates directly on the CSD and the correct kinetic parameters are involved thus it provides the "Optimal" solution. However, since the CSD is difficult to measure online and in real time the non-weighted CLD (NW CLD) based optimum temperature trajectory was also computed. The "NW CLD based optimum" curve has been calculated involving the "Plant parameters" based on the non-weighted CLD. As it can be seen, this curve, similarly to Figure $6 \mathrm{a}$, is above of the CSD based optimal temperature profile. This PMM situation is demonstrated by carrying out a CLD based optimization involving the "Model parameters", which is the "NW CLD based model profile". This differs significantly from both the CSD and the CLD based optimal temperature profiles and it gives the worst CSD. The "NW CLD based GHE+NMPC" is a control simulation, started with 
the "Model parameters" but the GHE continuously re-adjusts them, as it was described in the previous section. Very good agreement between the NMPC run and the CLD based optimal temperature profile can be achieved: these two cooling profiles practically overlap. The results indicate the robustness of GHE-NMPC combination against parametric PMM, ensured by the efficient state estimation and continuous model improvement.

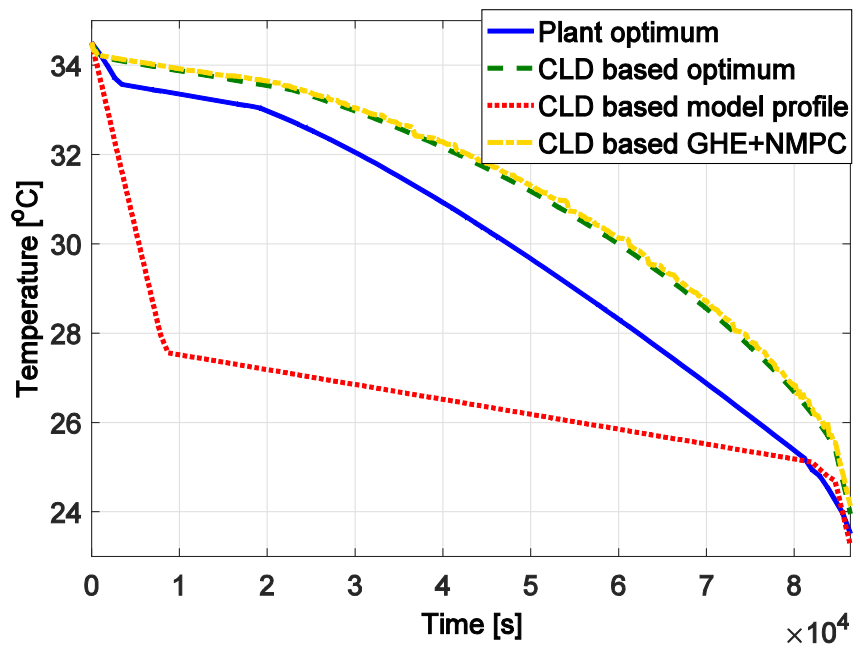

a)

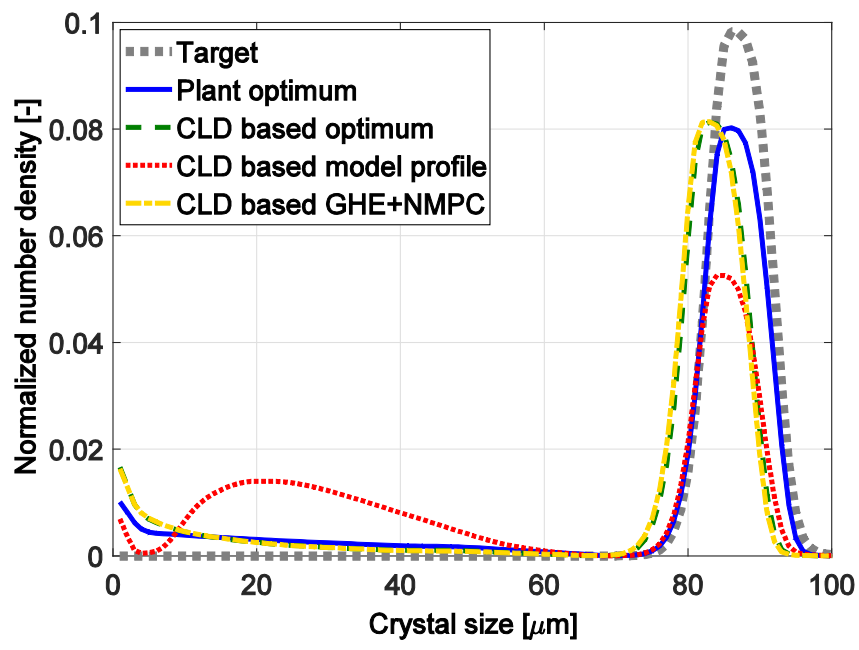

b)

Figure 8. The performance of RHE: a) the open loop optimal temperature profiles and the GHENMPC control and $b$ ) the corresponding product CSDs. 
Figure 9 illustrates the time evolution of the CSD during the GHE-NMPC run with temperature profile shown in Figure 8a. The optimal temperature profile creates supersaturation that causes a steady crystal growth while avoiding nucleation. As the amount of crystal increases the supersaturation is depleted faster hence the cooling can be accelerated as the batch progresses.

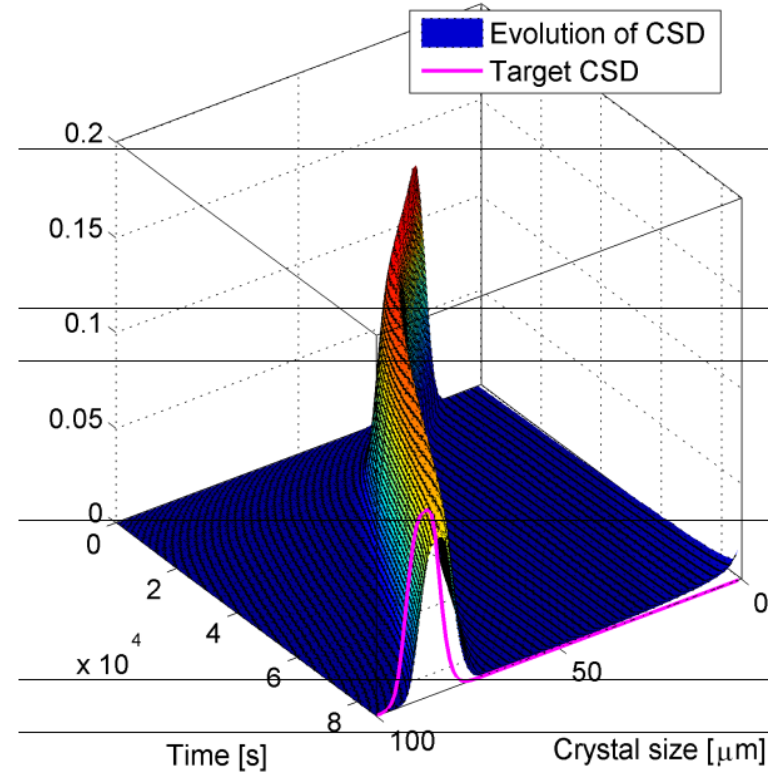

Figure 9. The dynamic evolution of the CSD during the GHE-NMPC batch.

According to the Figure $6 \mathrm{~b}$ and Figure 9, there is an offset between the target and achieved CSDs in the CLD based optimization. This is caused by the fact that the CLD based optimum temperature is suboptimal for the CSD, which is indirectly controlled through the CLD. A batch-to-batch improvement strategy might be applied in which an artificial offset in the CLD target can be reestimated after each batch based on end of batch CSD measurement and calculated CSD with the PBM. This approach will inherently lead to the reduction of the difference between the target and CSD.

Since both the NMPC and the GHE optimizations require considerable computational time, to achieve real time feasibility significant calculation speed-up is required, which can be achieved 
using a combination of efficient real-time iteration scheme and novel formulation of the GHENMPC approach.

\section{NMPC development and performance}

The significant calculation time requires the application of accelerated optimization techniques. In earlier studies the most common dynamic optimization algorithms have been compared ${ }^{24,40}$ and it was found that the direct optimization and multiple shooting over-performed the direct single shooting in computational time, however, these were more sensitive for premature stops thus in this work the direct single shooting method is applied.

Dividing the batch time into $K=400$ (yielding a sampling time of $\delta t=216 s$ ) leads to an optimization problem with 400 decision variables (which decreases as the batch time evolves). By applying the direct single shooting approach this optimization problem would hardly be solvable in real time, especially during the first part of the batch, until the number of decision variables reduces to a reasonable number. In this work a, computationally more efficient formulation of the direct single shooting is developed, based on the division of the original optimization to smaller sub-problems by grouping the decision variables. This strategy is referred to as "repeated sequential optimization" (RSO). As Figure 10 shows, the basic idea behind the RSO is to reduce the number of decision variables initially and solve more, but simpler optimization problems, gradually approaching towards the solution that is equivalent to the solution corresponding to the full discretization on the time scale according to $(\delta t)$. The first step is the calculation of a crude optimal temperature profile, using a fixed number of discretization $\left(n_{p, 1}\right)$. Hence the discretization time interval changes as the NMPC progresses during the batch. For the first (coarse) optimization stage an equally distributed discretization time is used, recalculated for each NMPC iteration $k$, as $\Delta t_{1, k}=\max \left(\delta t,\left(t_{K}-t_{k}\right) / n_{p, 1}\right)$. In this example a discretization of $n_{p, 1}=10$ was used. 
In the second step the crude optimal profile from the level 1 optimization is used as the initial point for a second optimization, involving more decision variables (but still much less than the original problem). In this example the discretization was doubled $\left(n_{p, 2}=20\right)$, halving the equally distributed time intervals, calculated similarly as before, as $\Delta t_{2, k}=\max \left(\delta t,\left(t_{K}-t_{k}\right) / n_{p, 2}\right)$. As the second optimization is started from the close proximity of optimum, despite of increased number of decision variables, it converges quickly. The last stage is a third optimization: only the first interval of the second ("refined") profile is divided according to the original sampling time $(\delta t)$. Up to 10 intervals with the actual sampling time $(\delta t)$ are used in this example. The rest of the first sampling time $\left(\Delta t_{2, k}-10 \delta t\right)$ is lumped in a single decision variable and together with the other $n_{p, 2}-1$ variables, corresponding to the other optimization time intervals, $\Delta t_{2, k}$, is solved in the third level optimization. This optimization also converges quickly due to the existence of a good starting point. In each optimization step the initial temperature profile is calculated by interpolating the results from the previous step. The first temperature profile is interpolated from the optimal profile of the previous NMPC iteration. Piecewise cubic Hermite type interpolations are applied, calling the pchip function of Matlab. Note that since the optimization discretization intervals are recalculated in each NMPC iteration and are gradually shrinking, when $\Delta t_{2, k}=\delta t$ the second optimization stage is practically the same as the third and when $\Delta t_{1, k}=\delta t$ is reached the number of optimization variables starts decreasing in a shrinking horizon approach and the three optimization levels practically collapse in the level three optimization problem only. 


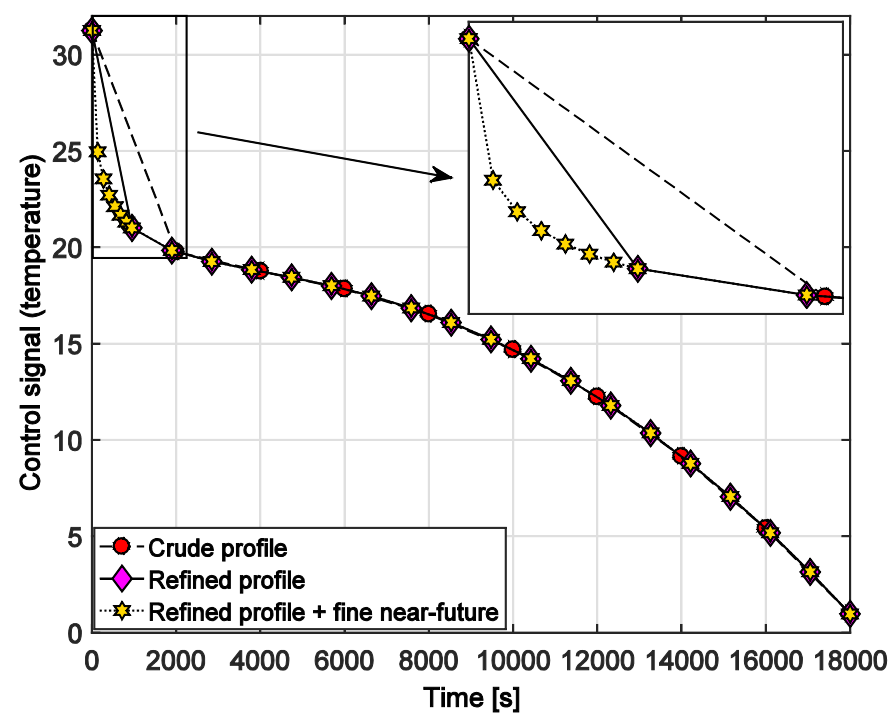

Figure 10. Schematic representation of the working principle of the three-stage repeated sequential optimization (RSO) strategy applied in the NMPC optimizations.

The RSO is a pseudo-warm start strategy, as the initial point is (nearly) optimal but the Jacobian needs to be recalculated. Also, the last step of the algorithm puts higher accent on the optimization of near-future temperatures, which is particularly useful since only the first temperature is implemented in every NMPC iteration.

The GHE/NMPC simulation, presented in Figure 7a was carried out with the RSO strategy, using the tuning parameters and constraints listed in Table 5. The achieved temperature profile is practically identical with the profile obtained with the full GHE/NMPC optimization of Figure 7a, which means that the RSO does not degrade the control quality.

Table 5. NMPC constraints and tuning parameters

\begin{tabular}{cll}
\hline Parameter & Name & Value/property \\
\hline $\boldsymbol{n}_{\boldsymbol{p}, \mathbf{1}}$ & Number of temperatures in first pre-optimization & 10 \\
$\boldsymbol{n}_{\boldsymbol{p}, \mathbf{2}}$ & Number of temperatures in second pre-optimization & 20 \\
$\boldsymbol{A}_{\boldsymbol{p}, \mathbf{1}}$ & Algorithm applied in first pre-optimization & Interior-point method
\end{tabular}



$\boldsymbol{A}_{\boldsymbol{p}, 2} \quad$ Algorithm applied in second pre-optimization
SQP
$\boldsymbol{A}_{\boldsymbol{p}, 3} \quad$ Algorithm applied in (third) optimization
SQP
$\boldsymbol{N}_{\boldsymbol{I} \mathbf{1}} \quad$ Maximum iteration in first pre-optimization
30
$\boldsymbol{N}_{\boldsymbol{I}, \mathbf{2}} \quad$ Maximum iteration in second pre-optimization
60
$\boldsymbol{N}_{\boldsymbol{I}, 3} \quad$ Maximum iteration in (third) optimization
100
$\boldsymbol{T}_{\boldsymbol{C}, \boldsymbol{m a x}} \quad$ Maximum cooling rate $\left[{ }^{\circ} \mathrm{C} / \mathrm{min}\right]$
0.5
$\boldsymbol{T}_{\boldsymbol{C}, \boldsymbol{m i n}} \quad$ Minimum cooling rate $\left[{ }^{\circ} \mathrm{C} / \mathrm{min}\right]$
0.01
$\boldsymbol{T}_{\boldsymbol{F}, \boldsymbol{m a x}} \quad$ Maximum final temperature $\left[{ }^{\circ} \mathrm{C}\right]$
$\boldsymbol{T}_{\boldsymbol{F}, \boldsymbol{m i n}} \quad$ Minimum final temperature $\left[{ }^{\circ} \mathrm{C}\right]$
18
$K \quad$ Number of discrete time moments in total batch time $\quad 400$

The calculation times for solving the GHE and NMPC problems corresponding to a GHE/NMPC simulation are shown in Figure 11. As expected, the GHE calculation time increases with the iterations due to expanding estimation horizon. Also, there is a decreasing tendency in the NMPC simulation time, but, due to the working principle of RSO this does no shows linear trend. Nevertheless, the total calculation time is well under the applied $216 s$ sampling time.

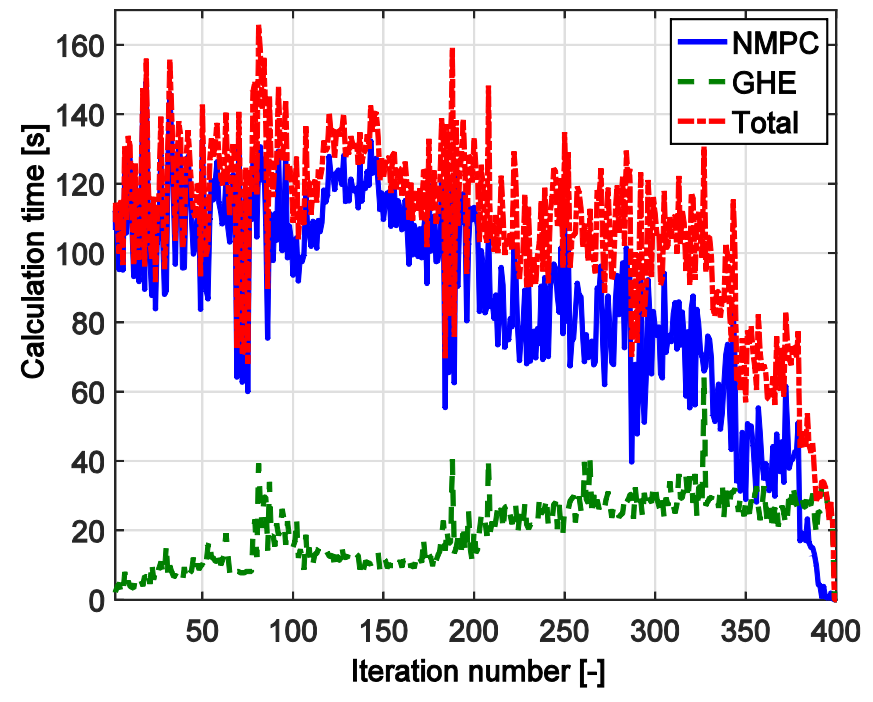


Figure 11. Calculations times for the solution of the GHE and NMPC problems in each control interval, obtained with $K=400$ (sampling time $=216 s$ ) using the RSO strategy. Calculation times obtained on an Intel i7-2600 3.4 GHz CPU, $1333 \mathrm{MHz}$ memory computer.

The calculation time is comparable with the sampling time thus this delay needs to be included into the NMPC algorithm. In the $j^{\text {th }}$ time moment the $(j+1)^{\text {th }}$ temperature must be readily computed since this has to be implemented in that moment to the real process. Due to the significant computation time, the calculation of the $(j+1)^{\text {th }}$ temperature need to be carried out in the $j^{\text {th }}-(j-1)^{\text {th }}$ time interval and must be finished (or stopped) at the latest when the process reaches the $j^{\text {th }}$ moment. However, the starting point of this optimization is the $j^{\text {th }}$ time moment. As a consequence, the initial states of NMPC optimizations are future states, which are supposed to be reached in the $j^{\text {th }}$ discrete time moment. These future states are estimated by the state estimator through process simulation. This strategy requires to find a tradeoff in choosing the best sampling time: applying higher sampling time reduces the probability of pre-mature optimization stops, hence the use of suboptimal control input, but on the other hand this might lead to less accurate state estimation, since the one step-ahead prediction of states may diverge more from the true value during a longer sampling period.

The effects of sampling time on the deviation from optimal NW CLD based temperature profile are depicted in Figure 12. According to Figure 11 the maximum calculation time is around $160 \mathrm{~s}$. These results confirm that the NMPC performance degrades if too low sampling time is applied, due to premature optimization stops, and also deteriorates with too high sampling times because of state estimation uncertainties. Of course, while computational restrictions represent a limitation, the selected sampling time must be in agreement with the process dynamics ${ }^{41}$. Taking into 
consideration the dynamics of industrial crystallizers $\delta t=144 s$ and $\delta t=216 s$ sampling times appear to be reasonable choices ${ }^{27}$.

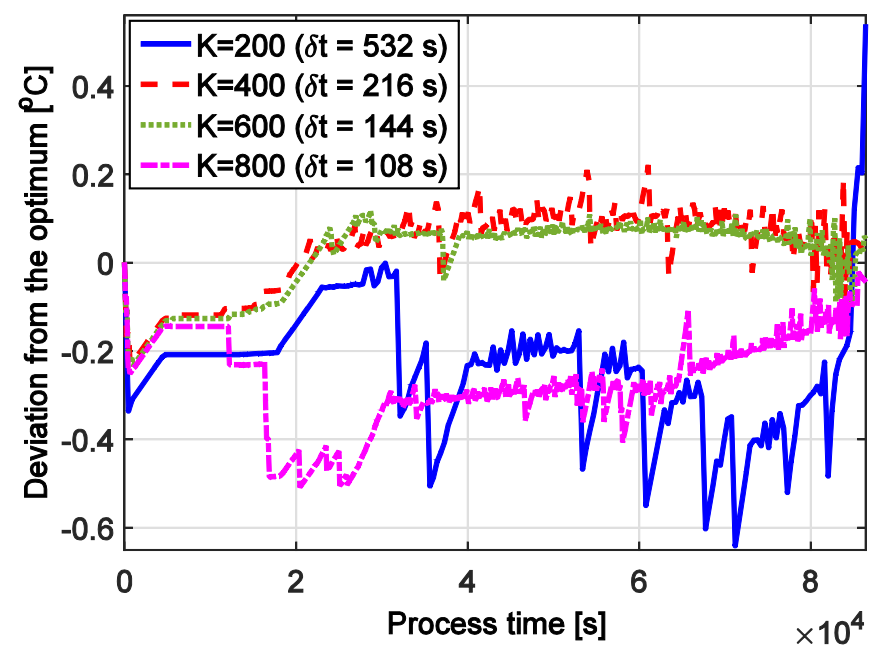

Figure 12. Performance of constrained GHE/NMPC with the included computational delay: effect of sampling time on deviation from the optimal temperature profile.

The GHE has the inherent property of mitigating the effects of disturbances. Although, if the measured signal is very noisy it might be meaningful to reduce the noise before using it in the control algorithm. Here an artificial random noise is applied on the "Plant" concentration data that often occurs in real systems, which is then reduced in the NMPC using a first order SavitzkyGolay filter ${ }^{42}$. Based on the results of preliminary simulations, $F=51$ frame length was applied in the filter. Comparison between the original, noisy and filtered concentrations is presented in Figure 13. It should be highlighted that using a proper calibration method and state-of-the-art spectroscopy tool, the measured concentration is significantly less noisy, thus it is more reliable for feedback control system. ${ }^{43}$ In this simulation we intentionally oversize the noise to push the system towards its limits. 


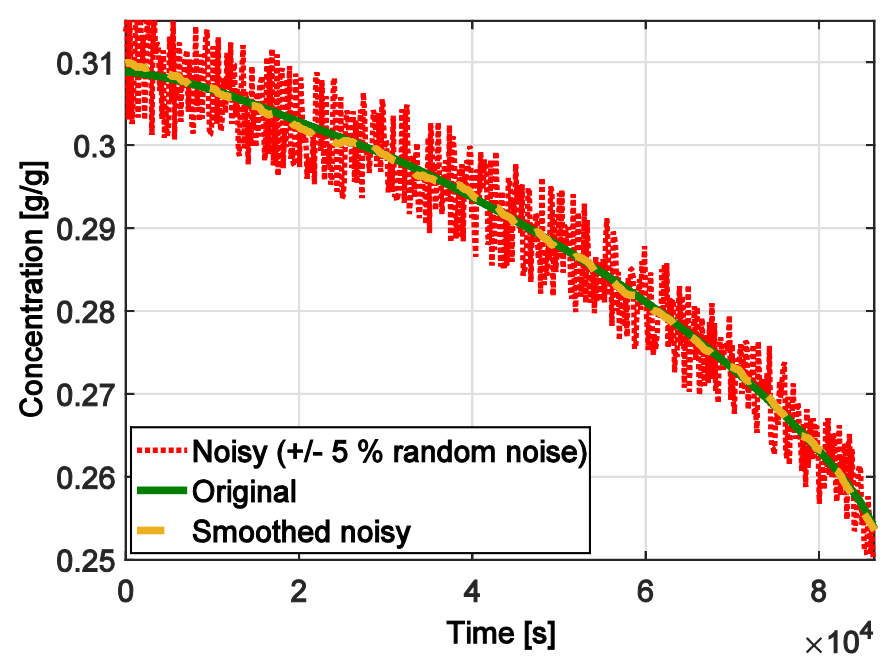

Figure 13. Original, noisy and smoothed (Savitzky-Golay filter) concentrations.

The results presented in Figure 14 indicate that the process behaves similarly with and without noise reduction. According to the simulations, the noisy concentration measurement translates to some noise in the implemented temperature profile, which, however, from practical aspects is negligible. As the figure shows, for noisy measurements the GHE should use more data points from the estimation horizon. Otherwise the effects of process noise can translate into poorly estimated kinetic constants from the GHE to NMPC, which significantly degrades the overall control performance.

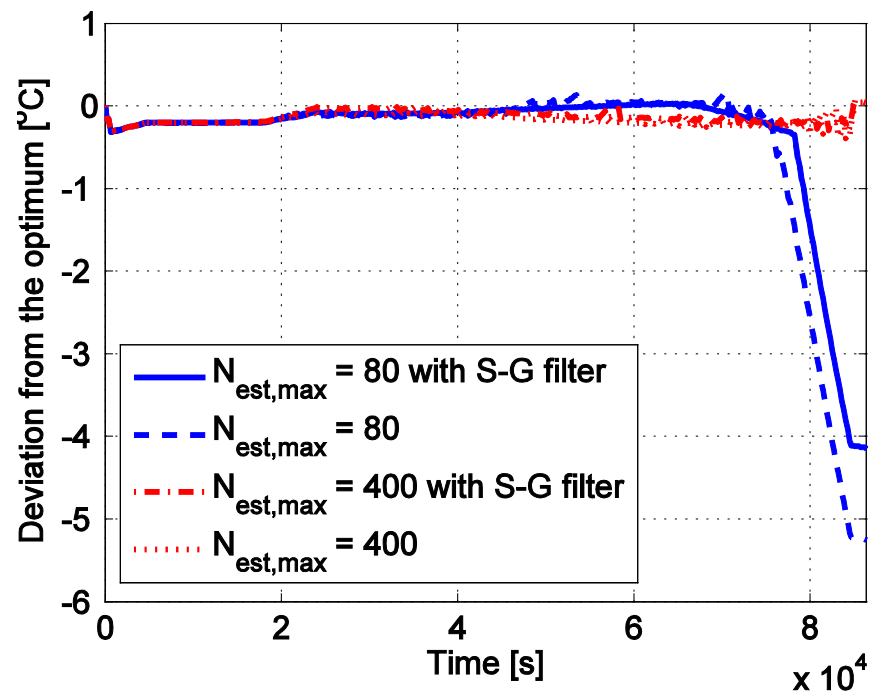


Figure 14. The control performance under plus-minus $5 \%$ random noise in concentration: effects of state estimator tuning $\left(N_{\text {est, } \max }\right)$ and the concentration smoothing.

It is remarkable that the last part of temperature profile with $N_{\text {est,max }}=400$ presents instabilities. This is explained by the premature optimization stops (see the timings of Figure 15). As it was highlighted before, the simulation time increases with the number of time moments in which the results are calculated and returned by the simulation function. The GHE calculation time increases with $N_{\text {est,max }}$. This leads to pre-mature NMPC optimization stops, which translates into temperature profile instabilities in the affected domains.

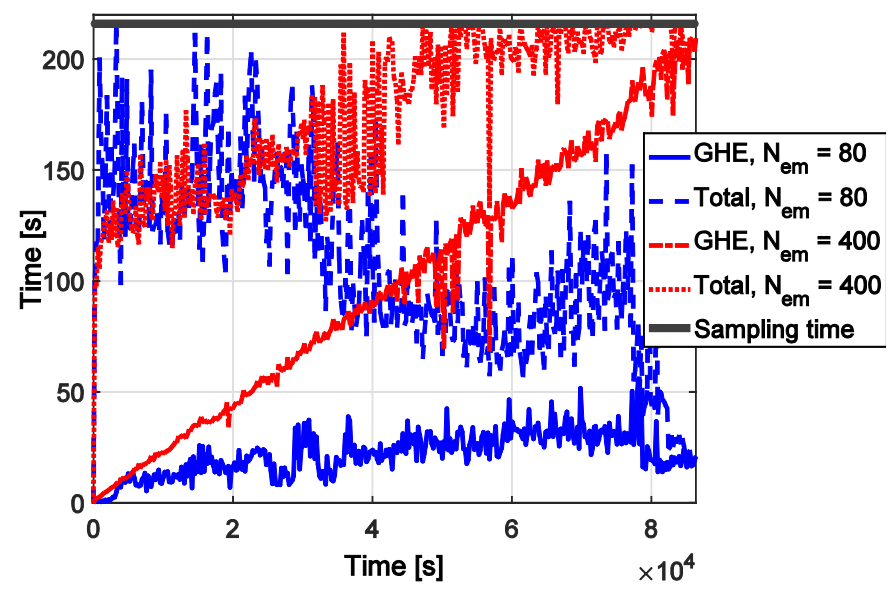

Figure 15. Calculation times with different GHE tuning, $K=400$. Intel i7-2600 $3.4 \mathrm{GHz} \mathrm{CPU}$, $1333 \mathrm{MHz}$ memory. $N_{e m}$ is $N_{\text {est, } \max }$.

\section{Conclusions}

In this work a feasible chord length distribution (CLD) based nonlinear model predictive control system (NMPC) was developed and presented involving high accuracy full population balance model $(\mathrm{PBM})$ based simulations. A fast CSD $\rightarrow$ CLD transformation has been developed, according to which the most probable CLD of individual crystals are calculated based on mapping the 
possible chord lengths of the cube shaped crystal. For the entire crystal population, the CLD is computed as the weighted sum of individual crystal CLDs.

The parametric plant model mismatch (PMM) is a general problem in NMPC development as the crystallization kinetics is system sensitive. In this study a growing horizon estimator (GHE) was employed, which provides an adaptive feature for the NMPC system, making it robust against PMM. In addition to the estimation of un-measurable system states (initial point of NMCP optimization), the GHE also has the role of model parameter re-adjustment. This strategy continuously improves the prediction accuracy of the model and it adapts to the process under PMM conditions.

To reduce the computational time an improved direct single shooting dynamic optimization algorithm was developed. The proposed repeated sequential optimization (RSO) approach improved significantly the calculation time with no effect on control performance. The required solution time (GHE+NMPC) is within the range of sampling times that would be suitable to use in the case of industrial crystallizers, indicating that the proposed efficient GHE/NMPC with realtime iteration scheme is ready for implementation in practical setup.

The GHE-NMPC was tested in simulations under parametric PMM conditions with significant concentration measurement noise. According to the results, good GHE tuning is a key for overall control system performance, and a well-tuned system is able to provide stable, high quality control even under realistic control conditions with practically relevant sampling time and PMM.

\section{Corresponding Author}

*zknagy@purdue.edu, z.k.nagy@lboro.ac.uk

\section{Acknowledgements}


The financial support of the International Fine Particle Research Institution is acknowledged gratefully. Funding from the European Research Council under the European Union's Seventh Frame-work Programme (FP7/2007-2013)/ERC grant agreement No. [280106-CrySys] is also acknowledged.

\section{Author Contributions}

The manuscript was written through contributions of all authors. All authors have given approval to the final version of the manuscript. All authors contributed equally.

\section{Abbreviations}

CLD, Chord Length Distribution; CSD, Crystal Size Distribution; DNC, Direct Nucleation Control; FBRM, Focused Beam Reflectance Measurement; HR-FVM, High Resolution Finite Volume Method; LW, Linearly Weighted (LCD); GHE, Growing Horizon Estimator; MPC, Model Predictive Control; NMPC, Nonlinear Model Predictive Control; NW, Non Weighted (CLD); PAT, Process Analytical Technology; PB, Population Balance; PBE, Population Balance Equation; PBM, Population Balance Model; PDE, Partial Differential Equation; PMM, Plant Model Mismatch; PVM, Process Vision Microscopy; RHE, Receding Horizon Estimator; RSO, Repeated Sequential Optimization; SSC, Supersaturation Control; SW, Square Weighted (CLD); SQP, Sequential Quadratic Programming;

\section{References}

(1) Mersmann, A. Crystallization Technology Handbook; Marcel Dekker Inc., New York, Basel, 2001.

(2) Myerson, A. S. Handbook of Industrial Crystallization; Elsevier, 2002.

(3) Tavare, N. S. Industrial Crystallization: Process Simulation Analysis and Design; The 
Plenum Chemical Engineering Series; Springer US, 1995.

(4) Nagy, Z. K.; Fevotte, G.; Kramer, H.; Simon, L. L. Recent Advances in the Monitoring, Modelling and Control of Crystallization Systems. Chem. Eng. Res. Des. 2013, 91 (10), 1903.

(5) Abu Bakar, M. R.; Nagy, Z. K.; Saleemi, A. N.; Rielly, C. D. The Impact of Direct Nucleation Control on Crystal Size Distribution in Pharmaceutical Crystallization Processes. Cryst. Growth Des. 2009, 9 (3), 1378.

(6) Nagy, Z. K.; Braatz, R. D. Advances and New Directions in Crystallization Control. Annu. Rev. Chem. Biomol. Eng. 2012, 3 (1), 55.

(7) Griffin, D. J.; Kawajiri, Y.; Grover, M. A.; Rousseau, R. W. Feedback Control of Multicomponent Salt Crystallization. Cryst. Growth Des. 2015, 15 (1), 305.

(8) Hermanto, M. W.; Chiu, M.-S. Sen; Braatz, R. D. Nonlinear Model Predictive Control for the Polymorphic Transformation of L-Glutamic Acid Crystals. AIChE J. 2009, 55 (10), 2631.

(9) Hulburt, H. M.; Katz, S. Some Problems in Particle Technology. A Statistical Mechanical Formulation. Chem. Eng. Sci. 1964, 19, 555.

(10) Samad, N. A. F. A.; Singh, R.; Sin, G.; Gernaey, K. V.; Gani, R. A Generic MultiDimensional Model-Based System for Batch Cooling Crystallization Processes. Comput. Chem. Eng. 2011, 35 (5), 828.

(11) LeVeque, R. J. Finite Volume Methods for Hyperbolic Problems; Cambridge university press, 2002; Vol. 31. 
(12) Mesbah, A.; Kramer, H. J. M.; Huesman, A. E. M.; Van den Hof, P. M. J. A Control Oriented Study on the Numerical Solution of the Population Balance Equation for Crystallization Processes. Chem. Eng. Sci. 2009, 64 (20), 4262.

(13) Randolph, A.; Larson, M. Theory of Particulate Processes, Analysis and Techniques of Continuous Crystallization; Academic Press: Salt Lake City, 1988.

(14) Ma, D. L.; Tafti, D. K.; Braatz, R. D. High-Resolution Simulation of Multidimensional Crystal Growth. Ind. Eng. Chem. Res. 2002, 41 (25), 6217.

(15) Szilágyi, B.; Nagy, Z. K. Graphical Processing Unit (GPU) Acceleration for Numerical Solution of Population Balance Models Using High Resolution Finite Volume Algorithm. Comput. Chem. Eng. 2016, 91, 167.

(16) Acevedo, D.; Tandy, Y.; Nagy, Z. K. Multiobjective Optimization of an Unseeded Batch Cooling Crystallizer for Shape and Size Manipulation. Ind. Eng. Chem. Res. 2015, 54 (7), 2156.

(17) Mesbah, A.; Landlust, J.; Huesman, A. E. M. E. M.; Kramer, H. J. M. J. M.; Jansens, P. J. J.; Van den Hof, P. M. J. M. J. A Model-Based Control Framework for Industrial Batch Crystallization Processes. Chem. Eng. Res. Des. 2010, 88 (9), 1223.

(18) Moldoványi, N.; Lakatos, B. G.; Szeifert, F. Model Predictive Control of \{MSMPR\} Crystallizers. J. Cryst. Growth 2005, 275 (1-2), e1349.

(19) Griffin, D. J.; Grover, M. A.; Kawajiri, Y.; Rousseau, R. W. Data-Driven Modeling and Dynamic Programming Applied to Batch Cooling Crystallization. Ind. Eng. Chem. Res. 2016, $55(5), 1361$. 
(20) Ma, C. Y.; Wang, X. Z. Model Identification of Crystal Facet Growth Kinetics in Morphological Population Balance Modeling of L-Glutamic Acid Crystallization and Experimental Validation. Chem. Eng. Sci. 2012, 70, 22.

(21) Simon, L. L.; Nagy, Z. K.; Hungerbuhler, K. Comparison of External Bulk Video Imaging with Focused Beam Reflectance Measurement and Ultra-Violet Visible Spectroscopy for Metastable Zone Identification in Food and Pharmaceutical Crystallization Processes. Chem. Eng. Sci. 2009, 64 (14), 3344.

(22) Simone, E.; Szilagyi, B.; Nagy, Z. K. K. Systematic Model Identification and OptimizationBased Active Polymorphic Control of Crystallization Processes. Chem. Eng. Sci. 2017, 174, 374.

(23) Ma, C. Y.; Wang, X. Z. Closed-Loop Control of Crystal Shape in Cooling Crystallization of L-Glutamic Acid. J. Process Control 2012, 22 (1), 72.

(24) Mesbah, A.; Nagy, Z. K.; Huesman, A. E. M.; Kramer, H. J. M.; Hof, P. M. J. Van den. Nonlinear Model-Based Control of a Semi-Industrial Batch Crystallizer Using a Population Balance Modeling Framework. IEEE Transactions on Control Systems Technology. 2012, pp 1188-1201.

(25) Mesbah, A.; Huesman, A. E. M.; Kramer, H. J. M.; Van den Hof, P. M. J. A Comparison of Nonlinear Observers for Output Feedback Model-Based Control of Seeded Batch Crystallization Processes. J. Process Control 2011, 21 (4), 652.

(26) Wan, E. A.; Merwe, R. Van Der. The Unscented Kalman Filter for Nonlinear Estimation. Adaptive Systems for Signal Processing, Communications, and Control Symposium 2000. 
AS-SPCC. The IEEE 2000. 2000, pp 153-158.

(27) Nagy, Z. K.; Braatz, R. D. Robust Nonlinear Model Predictive Control of Batch Processes. AIChE J. 2003, 49 (7), 1776.

(28) Motz, S.; Mannal, S.; Gilles, E. D. State Estimation in Batch Crystallization Using Reduced Population Models. J. Process Control 2008, 18 (3-4), 361.

(29) Kwon, J. S.-I. Il; Nayhouse, M.; Orkoulas, G.; Ni, D.; Christofides, P. D. A Method for Handling Batch-to-Batch Parametric Drift Using Moving Horizon Estimation: Application to Run-to-Run MPC of Batch Crystallization. Chem. Eng. Sci. 2015, 127, 210.

(30) Kail, N.; Marquardt, W.; Briesen, H. Estimation of Particle Size Distributions from Focused Beam Reflectance Measurements Based on an Optical Model. Chem. Eng. Sci. 2009, 64 (5), 984.

(31) Agimelen, O. S.; Hamilton, P.; Haley, I.; Nordon, A.; Vasile, M.; Sefcik, J.; Mulholland, A. J. Estimation of Particle Size Distribution and Aspect Ratio of Non-Spherical Particles from Chord Length Distribution. Chem. Eng. Sci. 2015, 123, 629.

(32) Hukkanen, E. J.; Braatz, R. D. Measurement of Particle Size Distribution in Suspension Polymerization Using in Situ Laser Backscattering. Sensors Actuators, B Chem. 2003, 96 (1-2), 451 .

(33) Li, M.; Wilkinson, D.; Patchigolla, K. Obtaining Particle Size Distribution from Chord Length Measurements. Part. Part. Syst. Charact. 2006, 23 (2), 170.

(34) Worlitschek, J.; Hocker, T.; Mazzotti, M. Restoration of PSD from Chord Length Distribution Data Using the Method of Projections onto Convex Sets. Part. Part. Syst. 
Charact. 2005, 22 (2), 81.

(35) Gunawan, R.; Fusman, I.; Braatz, R. D. High Resolution Algorithms for Multidimensional Population Balance Equations. AIChE J. 2004, 50 (11), 2738.

(36) Wynn, E. J. W. Relationship between Particle-Size and Chord-Length Distributions in Focused Beam Reflectance Measurement: Stability of Direct Inversion and Weighting. Powder Technology. 2003, pp 125-133.

(37) Beckmann, W. Crystallization: Basic Concepts and Industrial Applications; 2013.

(38) Lewis, R. M.; Shepherd, A.; Torczon, V. Implementing Generating Set Search Methods for Linearly Constrained Minimization. SIAM J. Sci. Comput. 2007, 29, 2507.

(39) Rawlings, J. B.; Miller, S. M.; Witkowski, W. R. Model Identification and Control of Solution Crystallization Processes: A Review. Ind. Eng. Chem. Res. 1993, 32 (7), 1275.

(40) Cao, Y.; Acevedo, D.; Nagy, Z. K.; Laird, C. D. Real-Time Feasible Multi-Objective Optimization Based Nonlinear Model Predictive Control of Particle Size and Shape in a Batch Crystallization Process. Control Eng. Pract. 2017, 69, 1.

(41) Candes, E. J.; Wakin, M. B. An Introduction To Compressive Sampling. IEEE Signal Process. Mag. 2008, 25 (2), 21.

(42) Savitzky, A.; Golay, M. J. E. Smoothing and Differentiation of Data by Simplified Least Squares Procedures. Anal. Chem. 1964, $36(8), 1627$.

(43) Griffin, D. J.; Grover, M. A.; Kawajiri, Y.; Rousseau, R. W. Combining ATR-FTIR and FBRM for Feedback on Crystal Size. In Proceedings of the American Control Conference; 
2015; Vol. 2015-July, pp 4308-4313. 